\title{
Huella hídrica y productividad económica del agua en Nogal Pecanero (Carya illinoiensis) al sur oeste de Coahuila, México *
}

\author{
JOSE LUIS RIOS-FLORES ${ }^{\text {a }}$, CAYETANO NAVARRETE-MOLINA ${ }^{\text {a }}$ \\ a Universidad Autónoma Chapingo, Unidad Regional Universitaria de Zonas Áridas, Campus \\ Central, Km.385 Carretera México-Texcoco, México. E-mail: j.rf2005@hotmail.com, \\ ingnavarretem@hotmail.com
}

\begin{abstract}
RESUMEN
Se determino la huella hídrica y la productividad económica del agua, el capital y la fuerza laboral en el cultivo de nogal pecanero (Carya illinoiensis) en Francisco I. Madero (FIM), Coahuila, México, y se comparó con los obtenidos a nivel Distrito de Riego 017 (DR017), Comarca Lagunera, México. Se utilizaron los modelos matemáticos de Ríos et. al., (2015, 2016, 2016a). Los resultados, al comparar FIM con el DR017, indican que la productividad del agua en términos físicos fue de $0,064 \mathrm{~kg} \mathrm{~m}^{-3}$ vs $0,075 \mathrm{~kg} \mathrm{~m}^{-3}$, indicando que FIM fue $16 \%$ menos productivo en el uso del agua. Asimismo el nogal empleó $15,73 \mathrm{~m}^{3} \mathrm{~kg}^{-1}$ vs $13,25 \mathrm{~m}^{3} \mathrm{~kg}^{-1}$ respectivamente. Un hm${ }^{3}$, produjo USD $10.921 \mathrm{de}$ ganancia y 17,00 empleos vs USD 86.178 y 14,70. El costo del agua al productor fue en extremo bajo: USD 0,0006 vs USD 0,0096. La Relación Beneficio/Costo fue 1,097 vs 2,138. La productividad del suelo fue de 0,94 ton ha' ${ }^{-1}$ vs 1,28 ton ha ${ }^{-1}$, mientras la ganancia por hectárea fue USD 161 vs USD 1.462. Un millón de USD de inversión de capital generó 151 empleos vs 194 empleos. Una hora de trabajo produjo 1,62 kg de nuez vs 2,23 kg de nuez, así como USD 0,28 y USD 2,55 de ganancia respectivamente, para todos los indicadores, en FIM y el DR017.
\end{abstract}

Palabras clave: Agua virtual, eficiencia del agua, nogal, productividad aparente del agua.

\section{Water Footprint and Economic Productivity of Water in Pecan (Carya Illinoiensis) at Southwestern Coahuila, México}

\begin{abstract}
The water footprint and economic productivity of water, capital and labor force were determined in pecan (Carya illinoiensis) cultivation in Francisco I. Madero (FIM), Coahuila, Mexico, and data gathered compared to that derived from Water District - 017 (WD-017) Comarca Lagunera, Mexico. They were used the mathematical models of Ríos et. al., (2015, 2016, 2016a). Results indicate that water production in physical terms; when comparing FIM with WD017 was $0,064 \mathrm{~kg} \mathrm{~m}^{-3}$ vs $0,075 \mathrm{~kg} \mathrm{~m}^{-3}$, show that FIM was $16 \%$ less productive in water use. Likewise, FIM pecans

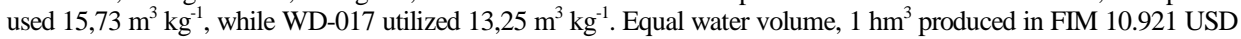
profits and 17 work spaces, and USD 86.178 USD and 14,70 jobs in WD-017. Producers water cost was extremely low: 0,0006 vs 0,0096 USD. The cost-benefit analysis in FIM and WD-017 was 1,097 and 2,138 respectively. Soil productivity was 0,94 ton ha ${ }^{-1}$ in FIM and 1,28 ton ha ${ }^{-1}$ in WD-017, while the gain ha ${ }^{-1}$ was 161 USD in FIM and 1.462 USD in WD-017. One million USD capital investment generated 151 work spaces in FIM and 194 in WD-017. One labor hour yielded 1,62 kg nuts in FIM and 2,23 kg at WD-017, as well as 0,28 and 2,55 USD profit for FIM and WD-017, respectively.
\end{abstract}

Keywords: Virtual Water, Water Efficiency, Pecan, Water Footprint.

Clasificación JEL: C3

\footnotetext{
* Los autores agradecemos sinceramente las sugerencias, observaciones y comentarios de los revisores que han venido a enriquecer el presente artículo.
}

Artículo recibido en mayo de 2017 y aceptado en agosto de 2017

Artículo disponible en versión electrónica en la página www.revista-eea.net, ref. ə-35312 


\section{INTODUCCIÓN}

El concepto de Huella hídrica fue introducido en respuesta a la necesidad de un indicador basado en el consumo del agua dulce (Hoekstra y Hung, 2005). La huella hídrica en general indica un uso del agua directo e indirecto de un productor o de un consumidor. Esta a su vez se subdivide en huella hídrica azul, que se refiere al consumo del agua superficial o subterránea, la huella hídrica verde se refiere al consumo del agua de lluvia almacenada en el suelo como humedad, y la huella hídrica gris, se refiere a la contaminada y es definida como el volumen de agua dulce que se requiere para asimilar la carga de contaminantes, basados en los estándares de calidad ambiental del agua existentes (Hoekstra, 2009).

La huella hídrica se puede calcular para un producto en particular, así como para cualquier grupo de consumidores (i.e. un individuo, ciudad, provincia, estado o nación) o productores (i.e. organización, empresas privadas, o sectores económicos) y es definida como el volumen total de agua dulce empleada para producir un bien o servicio consumido por un individuo o comunidad (Hoekstra y Chapagain, 2008).

Hoekstra y Chapagain (2004), mencionan que la producción de nuez de cáscara a nivel mundial utiliza el $2 \%$ del total del agua destinada para riego, al igual que la producción del café y del mijo, mientras que el maíz utiliza 9\% del total del agua. Wilchens, (2001), menciona que la producción de nuez con cáscara, en el caso de México, utiliza $2.811 \mathrm{~m}^{3}$ de agua $\mathrm{t}^{-1}$, mientras que el pistacho, que también es característico de la región utiliza $25.496 \mathrm{~m}^{3}$ de agua $\mathrm{t}^{-1}$, la naranja $772 \mathrm{~m}^{3}$ de agua $\mathrm{t}^{-1}$, las mandarinas, nectarinas y chabacanos $3.165 \mathrm{~m}^{3}$ de agua $\mathrm{t}^{-1}$, mientras que la vid consume $601 \mathrm{~m}^{3}$ de agua $\mathrm{t}^{-1}$, la sandía utiliza 236 $\mathrm{m}^{3}$ de agua $\mathrm{t}^{-1}$ y el melón $169 \mathrm{~m}^{3}$ de agua $\mathrm{t}^{-1}$ de producto (Hoekstra y Chapagain, 2004).

De acuerdo con Hoekstra y Hung (2005), existen diferencias entre países en cuanto a la eficiencia productiva del agua en el nogal, por ejemplo mientras que en México como ya se mencionó antes se utilizan $2.811 \mathrm{~m}^{3}$ de agua $\mathrm{t}^{-1}$ de nuez, al comparar contra los principales países productores de nuez de cáscara se observa que Australia utiliza $2.623 \mathrm{~m}^{3}$ de agua $\mathrm{t}^{-1}$ de nuez, Argentina $1.702 \mathrm{~m}^{3}$ de agua $\mathrm{t}^{-1}$ de nuez, Sudáfrica $2.759 \mathrm{~m}^{3} \mathrm{t}^{-1}$, Perú $2.077 \mathrm{~m}^{3} \mathrm{t}^{-1}$, Israel $949 \mathrm{~m}^{3} \mathrm{t}^{-1}$, Brasil 2.087 $\mathrm{m}^{3} \mathrm{t}^{-1}$, Egipto $2.122 \mathrm{~m}^{3} \mathrm{t}^{-1}$ y Estados Unidos $1.150 \mathrm{~m}^{3} \mathrm{t}^{-1}$. Por ello el objetivo de este trabajo fue la determinación de la huella hídrica y la productividad del capital y del trabajo en el cultivo del nogal pecanero (Carya illinoiensis) producido en FIM, Coahuila, municipio perteneciente al Distrito de Riego 017, Comarca Lagunera. 


\section{MATERIALES Y MÉTODOS}

\subsection{Localización del área de estudio}

De acuerdo con el INEGI (2015), Entre los paralelos $26^{\circ} 17^{\prime}$ y $26^{\circ} 38^{\prime}$ de latitud norte; los meridianos $103^{\circ} 18^{\prime}$ y $103^{\circ} 10^{\prime}$ de longitud oeste; altitud entre 400 y $2.000 \mathrm{~m}$. Colinda al norte con los municipios de Sierra Mojada y Cuatro Ciénegas; al este con los municipios de Cuatro Ciénegas y San Pedro; al sur con el municipio de Matamoros; al oeste con el estado de Durango. Ocupa el 1.8\% de la superficie del estado.

La temperatura promedio oscila entre los $14-20^{\circ} \mathrm{C}$, la precipitación varia de los 100 - 400 mm. El clima se clasifica como Seco semicálido (86\%) y Seco muy cálido y cálido (14\%). Mientras que el uso del suelo se clasifica de la siguiente forma: Agricultura (13\%) y zona urbana (0,4\%) Matorral (83\%), pastizal $(3,5 \%)$ y bosque $(0,1 \%)$ (INEGI, 2015).

\subsection{Fuentes de información}

Se utilizó la base de datos del SIAP (2014), empleándose de esa fuente datos de superficie cosechada (ha), producción física anual (ton), Valor Bruto de la Producción (VBP, en $\$$ miles de pesos), con ellos se generaron los datos de rendimiento físico "RF" anual (ton ha ${ }^{-1}$ ), precios medios rurales (\$ nominales ton $^{-1}$ ), y rendimiento monetario "RM” por hectárea (\$ nominales ha ${ }^{-1}$ ).

Los costos de producción por hectárea "C" se tomaron de los anuarios estadísticos de la producción agropecuaria de la Secretaria de Agricultura, Ganadería, Desarrollo Rural, Pesca y Alimentación (SAGARPA, 2014). Así, al RM se le restó "C” y se obtuvo la ganancia por ha. Así, se determinó la rentabilidad del cultivo usando la fórmula de la Relación Beneficio-Costo "RB/C", esto es: $\mathrm{RB} / \mathrm{C}=\mathrm{RM} / \mathrm{C}$.

El volumen "V" de agua usado en una hectárea por el cultivo se obtuvo multiplicando la lámina de riego "LR", que no es otra cosa más que la altura de la columna de agua que demanda el cultivo usual en la región, por 10.000 (los $\mathrm{m}^{2}$ de una ha), y para obtener todo el volumen de agua usado en toda la superficie cosechada, se multiplicó "V" por la superficie cosechada. La LR es un dato que es generado por las instituciones agrícolas encargadas de la generación de la tecnología agrícola del riego en los cultivos de un país, en el caso de México, ese organismo es el INIFAP (iniciales del Instituto Nacional de Investigaciones Forestales, Agrícolas y Pecuarias).

Se definió un empleo como el equivalente a la cantidad de jornadas que un ser humano promedio realiza en un año en condiciones promedio. De esa manera, se supuso que se trabajaba una jornada por día (entendiendo que una jornada son ocho horas de trabajo) durante seis días a la semana por 48 semanas al año, es decir: 6 jornadas por 48 semanas = 288 jornadas $=1$ empleo. Así, para 
obtener el número de empleos se multiplicó el total de jornales por hectárea que se invierten normalmente en el cultivo por la superficie cosechada, eso arrojó el total de jornadas que en un año se generaron, luego se le dividió entre 288 para obtener así el número de empleos generados.

La metodología utilizada en este estudio si bien es semejante a la utilizada por García et al. (2013) en lo relativo a la definición de lo que aquí se ha denominado un empleo permanente y que el autor denomina "una unidad de trabajo", no obstante, la misma metodología, se arrojan diferentes cantidades de horas de trabajo, por ejemplo, en este trabajo se ha definido que un empleo permanente equivale a 288 jornadas al año, equivalente a su vez a 2304 horas de trabajo al año, mientras que García et al. (2013) registra que una unidad de trabajo equivale a 1800 horas en Murcia, España y 1560 horas en Cerdeña, Italia. Lo anterior obedece a que en este trabajo se ha tomado como 288 jornadas año (igual a 6 jornadas por semana por 48 semanas al año; entendida una jornada de trabajo como igual a 8 horas) como un número dado, cuando en realidad debiera ser considerado como una ecuación, en la que una unidad de trabajo o empleo permanente es una variable dependiente " $y$ " que depende de tres variables independientes: la cantidad "X1" de semanas trabajadas normalmente en condiciones sociales promedio en la agricultura, la cantidad "X2" de jornadas "J" de trabajo laboradas por semana y finalmente la cantidad "X3"promedio social de horas de una jornada de trabajo, así: $\mathrm{y}=\mathrm{X} 1 * \mathrm{X} 2 * \mathrm{X} 3$, en este estudio se consideró que $\mathrm{X} 1=48$ semanas de trabajo por año, $\mathrm{X} 2=6$ días de trabajo por semana y X3= 8 horas de trabajo por jornada, por lo que, necesariamente diferirá el total de horas de trabajo que una unidad de trabajo o empleo permanente demanda si las condiciones de desarrollo tecnológico de la agricultura son diferentes, por ejemplo, en una región donde la agricultura está tecnológicamente desarrollada, un agricultor trabajará 6 horas diarias durante 5 días a la semana durante 46 semanas al año, así, el total de horas laboradas al año será igual a 1.380 horas.

\subsection{Ecuaciones matemáticas empleadas y variables}

\subsubsection{De productividad del suelo ${ }^{1}$ :}

Ya que dentro del cálculo de la variable dependiente denominada "Huella hídrica” (HH en lo sucesivo), intervienen las variables independientes rendimiento físico "RF" por hectárea y la ganancia bruta "gb" por hectárea, es necesario dejar claro el origen de estas variables de las que depende la $\mathrm{HH}$, explicándolas ahora en función de las variables de las que a su vez ellas dependen, como es el caso del RF, que depende de dos variables que actúan de

\footnotetext{
${ }^{1}$ Fuente de las ecuaciones matemáticas de productividad del suelo y del capital invertido: Ríos et al. (2016 a).
} 
manera independiente: la producción física y la superficie cosechada, o en el caso de la ganancia "gb" bruta por hectárea, que depende matemáticamente de tres variables que actúan de manera independiente: el rendimiento físico RF, el precio "p" por tonelada de producto agrícola y el costo "C" de producción por hectárea (que puede asumir la forma de coste de operación "CO" o coste neto "CN", dependiendo de si se consideran en su estructura conceptos como renta del suelo, amortización de capital, costes financieros, y depreciación de maquinaria y equipo). En forma esquemática, el organigrama de cada una de esas variables de productividad del suelo asume las formas siguientes:

a) Rendimiento físico "RF", medido en ton $\mathrm{ha}^{-1}$, o en $\mathrm{kg}$ ha, según se lo demande en la ecuación pertinente, el RF está determinado por la ecuación:

$$
\mathrm{RF}=\frac{\text { Producción }}{\text { superficie cosechada }}=\frac{\text { ton de producto }}{\text { una ha }}=\frac{\mathrm{kg} \text { de producto por ha }}{\text { una ha }}
$$

\subsubsection{De la productividad del capital invertido:}

Marco teórico sobre la productividad del capital. La productividad del capital asume diversas formas, la más conocida es la rentabilidad financiera, que evaluada mediante el índice de la Relación Beneficio-Costo, señala, mediante un indicador mayor, menor o igual a la unidad si la inversión de capital es productiva (cuando el índice de la $\mathrm{RB} / \mathrm{C}>1$ ) o es improductiva (cuando la $\mathrm{RB} / \mathrm{C}<1$ ), no obstante, existen otros indicadores de la productividad del capital importantes desde el punto de vista social, por ello se le denomina productividad social del capital, ya que con un simple índice numérico se tiene idea de si es o no productivo socialmente, es el caso del indicador que señala cuántos empleos genera en al menos dos diferentes ramas productivas el mismo monto de inversión de capital. Una tercera forma de productividad del capital es aquella que señala, mediante un número indicador de índole marginalista, el coste por unidad de producto.

a) Ingreso por ha, llamado también rendimiento monetario "RM", medido en USD, determinado por la ecuación:

$$
\mathrm{RM}=R F^{*} p
$$

Donde;

$\mathrm{p}=$ precio ton $^{-1}$, en USD.

b) Ganancia Bruta por ha "gb", medida en USD, determinado por la ecuación:

$$
\mathrm{gb}=R M-C O
$$

Donde;

“CO” es el costo de operación por ha en USD.

c) Ganancia Neta por ha "gn”, medida en USD, determinado por la ecuación: 


$$
\text { gn }=R M-C N
$$

Donde:

“CN" es el costo total por ha en USD, donde C incluye depreciación de capital fijo y renta del suelo.

d) Relación Beneficio - Costo "RB/CN", donde "RB” es lo señalado arriba como "RM", esto es el ingreso por hectárea, mientras que "CN" es el costo neto. También se puede considerar al costo bruto "CB" en vez de $\mathrm{CN}$, ello implicaría que la $\mathrm{RB} / \mathrm{C}$ estaría saliendo artificialmente alta, es decir, estimar la $\mathrm{RB} / \mathrm{C}$ con $\mathrm{CN}$ es más exacta en tanto el costo por hectárea es más real, más elevado que CB. Se le estimó con el modelo

$$
\mathrm{RB} / \mathrm{C}=\frac{R M}{C N}
$$

e) La productividad social del capital puede cristalizar en diversas formas, pero en este trabajo se la entiende como la capacidad que tiene la inversión de capital en una determinada esfera productiva para la generación de una determinada cantidad de empleo, así, por ejemplo, si un mismo monto de capital, un millón de pesos, invertido en la producción de nogal genera 2 empleos mientras que ese mismo monto de inversión, si se lo invierte en la producción de manzana es capaz de generar 3 empleos, entonces, en la producción de manzana, el capital será 50\% más productivo socialmente que en la producción de nogal. La Productividad social del capital fue denominada con las siglas "Empleos MUSD-1", se mide como la cantidad de empleos "E" generados por cada millón de dólares invertidos en la producción, donde MUSD son las iniciales de millones de dólares norteamericanos invertidos en la producción, la productividad social del capital fue determinado por la ecuación:

$$
\mathrm{E} \text { MUSD }=\frac{31.250}{9}\left(\frac{J}{C N}\right)
$$

Donde;

"CN" es el costo neto por hectárea, es decir aquel costo que ya tomó en cuenta a la depreciación de maquinaria y equipo y la renta del suelo, puede también tomarse el costo bruto.

"CB" o costo de operación (esto es, el costo por hectárea que aún no considera la depreciación de maquinaria y equipo ni la renta del suelo), pero en tanto $\mathrm{CB}$ es mayor que $\mathrm{CN}$, necesariamente "E MUSD" será menor.

Otra forma de productividad social del capital, es aquella que mide a la cantidad de capital invertido necesario para crear un empleo permanente, llamémosle "USD E E ", el cual está dado por la ecuación: 


$$
\mathrm{USD} \mathrm{E}^{-1}=288 *\left(\frac{C N}{J}\right)
$$

\subsubsection{De la Huella Hídrica²:}

a) Huella hídrica en términos físicos (HHf1), como índice de productividad física del agua, expresada en $\mathrm{kg} \mathrm{m}^{-3}$, determinado por la ecuación

$$
\mathrm{HHf} 1=\frac{R F}{V}=\frac{R F}{10,000 L R}=0.0001 R F(L R)^{-1}
$$

Donde "V" es el volumen de agua usado por ha (en $\mathrm{m}^{3}$ ), equivalente al producto de la lámina de riego "LR" por $10 \mathrm{mil} \mathrm{m}$ '.

b) Huella hídrica en términos físicos (HHf2) como índice de eficiencia física del uso del agua, expresada en $\mathrm{m}^{3} \mathrm{~kg}^{-1}$, determinado por la ecuación:

$$
\text { HHF2 }=\frac{V}{R F}=\frac{10,000 L R}{R F}=10,000 L R(R F)^{-1}
$$

Donde;

RF está en $\mathrm{kg} \mathrm{ha}^{-1}$.

La metodología usada en este trabajo para determinar la $\mathrm{HH}$ física $\mathrm{y}$ económica es de naturaleza explícita, ya que Ríos et al. (2015, 2016 y 2016 a) señalan claramente en sus modelos matemáticos a las variables independientes de las que dependen y como actúa cada una de ellas, mientras que para otros autores al determinar la huella hídrica usan modelos matemáticos implícitos, en los que no aparece detallada, explícita, la ecuación donde se señalen todas y cada una de las variables independientes ni la forma en que actúa cada una de ellas en la ecuación correspondiente, basta observar el modelo matemático de Mekonnen y Hoekstra (2010) que permite estimar la huella hídrica física de un animal de granja, donde si bien la huella hídrica "WF" depende del agua virtual consumida en la alimentación "a”, así como la consumida "c" en forma de bebida y la que se usó en servicios "s”, no se explicita en la ecuación la regla de correspondencia entre cada una de las variables:

$$
W F_{\text {feed }}(a, c, s)=\frac{\sum_{p=1}^{n}\left(\text { Feed }(a, c, s) \times W F_{\text {mixing }}(a, c, s)\right.}{\operatorname{Pop}^{*}(a, c, s)}
$$

Así, las ventajas de un modelo explícito como el usado en este trabajo son varias, en primer lugar la certeza de saber el origen de lo que arroja el

\footnotetext{
${ }^{2}$ Fuente de los modelos matemáticos de huella hídrica: Ríos et al., 2015, 2016 y 2016 a.
} 
modelo de salida en función de los datos de entrada, así como la replicabilidad en otras condiciones diferentes a las de este estudio.

c) Huella hídrica en términos económicos (HHec1) como índice de productividad económica del agua usada en el riego, expresada en USD de ganancia bruta $\mathrm{hm}^{-3}$, determinada por la ecuación:

$$
H H_{e c 1}=\frac{g b}{V}=\frac{R F(p)-C}{10000 L R}
$$

d) Huella hídrica en términos económicos (HHec2) como índice de eficiencia económica del agua usada en el riego, sus unidades de expresión son $\mathrm{m}^{3}$ por unidad de ganancia bruta producida, y está determinada por la ecuación:

$$
H H_{e c 2}=\frac{V}{g b}=\frac{10000 L R}{R F(p)-C}
$$

Donde;

La ganancia bruta "gb" está en USD por ha, "V" en $\mathrm{m}^{3}$ por ha, "RF" es el rendimiento físico por ha, "p" es el precio por ton y "C" es el coste de producción por hectárea.

e) Huella hídrica en términos sociales (HHsoc1) como índice de productividad social, medida como la cantidad de empleos asociados al uso de un $\mathrm{hm}^{3}$ de agua usada en el riego, es decir: "Empleos $\mathrm{hm}^{-3}$," determinada por la ecuación:

$$
H H_{\text {soc1 }}=\frac{1000000(\mathrm{~J} / 288)}{V}=\frac{1000000(\mathrm{~J} / 288)}{10000 L R}=\frac{25}{72}\left(\frac{\mathrm{J}}{L R}\right)
$$

Donde "J" es el número de jornales por ha y 288 es el número de jornadas de trabajo que en un año tiene un trabajador a razón de 6 jornadas por semana durante 48 semanas al año. En lo relativo a la metodología de cálculo de la huella hídrica económica y social, existe semejanza con García et al. (2013), ya que ellos determinan la ganancia utilizando el precio promedio, igual que en este trabajo, en lo que existe diferencia metodológico-conceptual con García et al. (2013) es que ellos manejan la eficiencia del agua de riego tal como en este trabajo se le ha llamado índice de productividad social: cantidad de producto (ganancia si es económico o empleo si es social) por $\mathrm{m}^{3} \mathrm{o} \mathrm{hm^{3 }}$ según sea el caso. Ellos le llaman "Importancia social del agua de riego", mientras que en este trabajo se la ha llamado "Productividad social del agua de riego".

f) Huella hídrica en términos sociales (HHsoc2) como índice de eficiencia social del agua usada en el riego, medida como la cantidad de agua usada 
en la producción necesaria para generar un empleo permanente, medida en $\mathrm{m}^{3}$ empleo ${ }^{-1}$, determinada por la ecuación:

$$
H H_{\text {soc } 2}=\frac{V}{J / 288}==288 *\left(\frac{V}{J}\right)=288 *\left(\frac{10000 L R}{J}\right)
$$

Donde “J” es el número de jornales por ha y 288 es el número de jornadas de trabajo que en un año tiene un trabajador a razón de 6 jornadas por semana durante 48 semanas al año.

g) Precio del agua "Pagua", determinado por el precio estimado por del $\mathrm{m}^{3}$ de agua usada por el productor en el riego, medida en MX\$ o USD por $\mathrm{m}^{3}$, determinado por la ecuación:

$$
\mathrm{P}_{\text {agua }}=\frac{\text { costo del rubro del agua por ha }}{V}
$$

Si bien el marco teórico del significado de la huella hídrica fue ya señalado en la parte de introducción, el procedimiento metodológico seguido en sus estimación debe ser explícito, por lo que, para una mejor lectura en el cálculo de la huella hídrica $(\mathrm{HH})$, ya sea ésta de índole física $\left(\mathrm{m}^{3} \mathrm{~kg}^{-1}\right)$, económica (MX\$ de ganancia $\mathrm{hm}^{-3}$ ) o social (Empleos $\mathrm{hm}^{-3}$ ), en forma sintética, se siguen los siguientes pasos:

Paso 1: Tener en claro que la $\mathrm{HH}$ es el resultado de la división de un cociente, de un número " $A$ " en el numerador entre un número "B" en el denominador.

Paso 2: Sí "A” es la cantidad de agua usada por hectárea en la producción agrícola $\left(\mathrm{m}^{3}\right)$ y "B" es la cantidad de producto físico (ton de producto agrícola por hectárea) o económico (cantidad de ganancia obtenida por hectárea) o social (cantidad de empleo asociado al uso de un $\mathrm{hm}^{3}$ de agua usada en el riego), entonces, el resultado de dividir A/B arrojará un número índice que mide la eficiencia con que se usa el agua de riego, ya que señalará cuánta $\left(\mathrm{m}^{3}, \mathrm{hm}^{3}\right.$, según se desee) agua se usó por unidad de producto físico (kg, ton, según se desee), o económico (unidades monetaria de ganancia) o social (por empleo permanente generado), quedando de esa manera expresada la $\mathrm{HH}$ física en $\mathrm{m}^{3}$ $\mathrm{kg}^{-1}$, la HH económica en $\mathrm{m}^{3}$ por unidad monetaria de ganancia, y finalmente la $\mathrm{HH}$ social en $\mathrm{m}^{3}$ empleo ${ }^{-1}$.

Paso 3: Sí “A” es la cantidad de agua usada por hectárea en la producción agrícola $\left(\mathrm{m}^{3}\right)$ y "B" es la cantidad de producto físico (ton de producto agrícola por hectárea) o económico (cantidad de ganancia obtenida por hectárea) o social (cantidad de empleo asociado al uso de un $\mathrm{hm}^{3}$ de agua usada en el riego), entonces, el resultado de dividir B/A arrojará un número índice que mide la productividad del agua de riego, ya que señalará la cantidad de producto (físico, económico o social) generado por unidad de volumen de agua $\left(\mathrm{m}^{3}, \mathrm{hm}^{3}\right.$, según se desee) usada en la producción, quedando de esa manera expresada la $\mathrm{HH}$ 
física en $\mathrm{kg} \mathrm{m}^{-3}$, la $\mathrm{HH}$ económica en ganancia $\mathrm{m}^{-3}$ o ganancia $\mathrm{hm}^{-3}, \mathrm{y}$ finalmente la $\mathrm{HH}$ social se expresará, bajo la forma de un índice de productividad $^{3}$ como empleos $\mathrm{hm}^{-3}$.

Paso 4: Para el cálculo de la HH física, económica o social, solamente se necesita conocer cada una de las variables señaladas en las ecuaciones 8 a 12, para así introducirlas a la ecuación correspondiente y de esa manera obtener de salida la correspondiente HH física, económica o social.

\subsubsection{De la productividad de la fuerza laboral:}

a) La primera forma en cómo se midió la productividad del trabajo fue en su forma de $\boldsymbol{k g}$ de nogal producidos por cada trabajador, a la que se representó como "KgT”, se le midió como Kg trabajador ${ }^{-1}$, se le determinó por la ecuación:

$$
K g T=288 *\left(\frac{R F}{j}\right)
$$

Donde:

RF debe estar en kg por hectárea, si se lo expresa en ton por hectárea la ecuación deberá multiplicarse por 1000.

b) La segunda forma en cómo se midió la productividad del trabajo se la representó con las literales “Kgh”, se le midió como la cantidad de nogal producido, en kg, producidos por hora de trabajo, con las unidades "Kg $\mathrm{h}^{-1}$,, determinado por la ecuación:

$$
K g h=125 *\left(\frac{R F}{J}\right)
$$

Donde:

RF debe estar en ton por hectárea, si se lo expresa en kg por hectárea la ecuación deberá dividirse entre 1000 .

c) La tercera forma en cómo se midió la productividad del trabajo, se le denominó "hton", fue en su forma de horas invertidas de trabajo por ton de nogal producido, medido con las unidades " $\mathbf{h} \mathbf{t o n}^{-1}$ ", determinado por la ecuación:

$$
\text { hton }=8 \frac{J}{R F}
$$

\footnotetext{
${ }^{3}$ Es de más fácil lectura un indicador de la $\mathrm{HH}$ social expresada como "empleos $\mathrm{hm}^{-3}$ " que en su

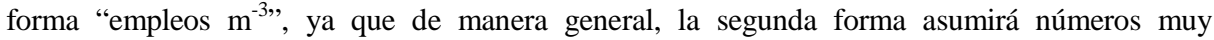
pequeños, por ejemplo en el caso del trigo en el norte de México, se tiene una $\mathrm{HH}$ social de 0.00000396 empleos $\mathrm{m}^{-3}$, que al ser expresado de la primer forma quedaría 3.96 empleos $\mathrm{hm}^{-3}$.
} 
Donde:

RF debe estar en ton por hectárea.

\section{RESULTADOS Y DISCUSIÓN}

Los indicadores de productividad y eficiencia mediante los cuales se evaluó a la huella hídrica, muestran en la Tabla 1. En esa fuente se observa que la productividad física de FIM, Coahuila en promedio fue de $0,064 \mathrm{~kg} \mathrm{~m}^{-3}$ (oscilando de $0,063 \mathrm{~kg} \mathrm{~m}^{-3}$ en nogal de bombeo a $0,070 \mathrm{~kg} \mathrm{~m}^{-3}$ en nogal de gravedad), mientras que a nivel distrital el indicador fue $0,075 \mathrm{~kg} \mathrm{~m}^{-3}$, lo que indica que la productividad física determinada en FIM, Coahuila fue 16\% inferior en comparación a la determinada a nivel distrital.

Visto desde otro ángulo, en FIM, Coahuila se requirieron un total de 15,73 $\mathrm{m}^{3} \mathrm{~kg}^{-1}$ de nuez $\left(15,78 \mathrm{~m}^{3} \mathrm{~kg}^{-1}\right.$ en riego por bombeo y $14,35 \mathrm{~m}^{3} \mathrm{~kg}^{-1}$ en riego por gravedad), mientras que a nivel Distrito de Riego, el indicador fue $13,25 \mathrm{~m}^{3} \mathrm{~kg}^{-1}$ de nuez, lo que indica que la productividad física medida en su forma $\mathrm{m}^{3} \mathrm{~kg}^{-1} \mathrm{de}$ FIM, Coahuila fue menor en relación al distrital, toda vez que empleo 19\% más del agua que se requirió a nivel distrital para generar ese mismo kilogramo de nuez. Hoekstra y Chapagain (2004) señalan que el promedio mundial de la huella hídrica azul es de $9,0 \mathrm{~m}^{3} \mathrm{~kg}^{-1}$, por lo que, con $15,75 \mathrm{~m}^{3} \mathrm{~kg}^{-1}$ de nuez se encuentra muy por encima, $75 \%$, de ese promedio mundial, lo que estaría señalando que debe mejorarse el uso del agua en nogal en el Sur Oeste de Coahuila, la mejoría comprende desde las prácticas de manejo hasta la mejora tecnológica de los sistemas de riego, ya que el riego actual usa el agua en forma poco eficiente, inundando los predios nogaleros, como si el agua fuese un recurso infinito.

Estos datos nos indican la gran cantidad de agua que requiere el nogal para transformarla en nuez, lo que nos muestra la importancia que tiene la determinación de este tipo de indicadores. El uso eficiente del agua es uno de los índices más ampliamente empleados en una gran variedad de cultivos en España (García et al., 2013), sin embargo en México existe muy poca información y en algunos cultivos nula información al respecto.

La segunda forma de evaluar a la huella hídrica fue como un indicador de productividad marginal en su aspecto económico, medido como USD de ganancia por $\mathrm{Hm}^{3}$ de agua usada en el riego, de esa forma, la Tabla 1 muestra que a nivel de agregación general, para FIM, Coahuila, se observó en principio que la relación entre volumen de agua irrigado y la ganancia bruta generada fue positivo, de USD 10.921 por hectómetro cúbico empleado en el riego (USD $9.270 \mathrm{hm}^{-3}$ en nogal de riego bombeo y USD $57.729 \mathrm{hm}^{-3}$ en nogal de riego por gravedad), mientras a nivel Distrito de Riego se obtuvieron USD 53.930 de ganancia por $\mathrm{hm}^{3}$, lo que indica que la ganancia bruta generada por hectómetro 
cúbico empleado en el riego de nogal fue inferior en FIM, Coahuila, al generar solamente un $20 \%$ de ganancia que se generó a nivel distrital.

Finalmente como otra forma de productividad se determinó la huella hídrica en su forma social, medida bajo la forma de empleos generados por cada $\mathrm{hm}^{3}$ de agua usada en el riego, a este respecto el Cuadro 1 señala que al emplearse distintas cantidades de jornales por hectárea 72,16 jornales ha ${ }^{-1}$ (72,27 jornales ha $^{-1}$ bombeo y 71,27 jornales ha $^{-1}$ en gravedad), mientras a nivel distrital se emplearon un total de 71,78 jornales $\mathrm{ha}^{-1}$, lo que indica que el municipio de FIM, Coahuila empleó en promedio 16\% más jornales por hectárea. Asimismo a nivel del municipio de FIM, Coahuila en promedio se generaron 17 empleos $\mathrm{hm}^{-3}$ (17,1 empleos hm ${ }^{-3}$ en nogal irrigado por bombeo y 13,6 empleos $\mathrm{hm}^{-3}$ en nogal irrigado por gravedad), mientras que a nivel Distrito de Riego se generaron 14,7 empleos $\mathrm{hm}^{-3}$, lo que indica que en términos sociales FIM, Coahuila fue más productivo socialmente al usar el agua de riego, ya que produjo 16\% más empleo por $\mathrm{hm}^{3}$ de agua irrigada que a nivel del DR-017

Es importante mencionar que este indicador es alto en relación a otros cultivos como los forrajes que emplean menos mano de obra durante sus procesos productivos, mientras que las hortalizas y los frutales obtienen un indicador más elevado. En este sentido, García et al. (2013), determinaron un índice que oscilo entre 24 - 62 empleos $\mathrm{hm}^{-3}$ en la producción de hortalizas y frutales, mientras que la producción de cultivos en invernadero generan hasta 190 empleos $\mathrm{hm}^{-3}$, asimismo Ríos et al. (2015), determinaron un promedio para cultivos forrajeros en la Comarca Lagunera de 0,048 empleos $\mathrm{hm}^{-3}$, con el que se muestra la importancia social que tiene la producción de nogal pecanero para el Distrito de Riego 017 y para el municipio de FIM, Coahuila en particular.

De la Tabla 1 se observa que el costo por metro cúbico de agua para riego del cultivo fue muy pequeño: 1,19 centavos de USD por $\mathrm{m}^{3}$ en FIM y 0,76 centavos de USD por $\mathrm{m}^{3}$ en promedio para todo el DR-017. Se dice es muy bajo el precio del agua al comparársele con lo encontrado por algunos autores, como Gleick (2000), quien indica que para el caso de los agricultores de Estados Unidos, determinó pagan USD $0,05 \mathrm{~m}^{3}$ (5 centavos de dólar por metro cúbico) empleado en el riego, mientras que el sector público de ese país, se paga de USD $0,30 \mathrm{~m}^{3}$ a USD $0,80 \mathrm{~m}^{3}$ de agua tratada para uso personal, es decir, de 30 a 80 centavos de USD por $\mathrm{m}^{3}$.

De acuerdo con Takele y Kallenbach (2001), los precios del agua son importantes para la mejora de la demanda y de la conservación de este recurso sin embargo a nivel mundial también existen ejemplos de que el recurso no se valora como recurso finito. Murphy (2003), determinó que los agricultores del Distrito de Riego en el Valle Imperial de California pagan solamente USD 15,50 por $1.200 \mathrm{~m}^{3}$, es decir, 1,2 centavos de USD por $\mathrm{m}^{3}$, coincidiendo plenamente con los 1,19 centavos de USD señalado en nuestra Tabla 1, coincidiendo también con lo 
encontrado por Ríos et al. (2015), quienes determinaron un precio promedio del agua igual a USD $0,02 \mathrm{~m}^{-3}$ (es decir, 2 centavos de USD por $\mathrm{m}^{3}$ ) para cultivos forrajeros producidos en el Distrito de Riego 017, mientras que en el Valle de Mexicali, Ríos et al. (2016a), determinaron un precio promedio del agua de riego igual a $\$ 0,19 \mathrm{~m}^{-3}$, es decir, 19 centavos de USD por $\mathrm{m}^{3}$.

\subsection{Productividad del capital}

La Tabla 1 muestra que el invertir un millón de USD en la producción de nogal pecanero, generó diferente cantidad de empleos en cada uno de los dos tipos de riego, mientras a nivel municipal se generaron 151,0 empleos por cada millón de USD invertido en la producción de nogal, desglosando esas cifras se observa que en el riego por bombeo se generaron 150,5 empleos, y en el riego por gravedad se generaron 175,1 empleos por cada millón de USD invertidos en la producción de nogal, mientras que a nivel Distrito de Riego 017 se generaron 194 empleos por cada millón de dólares norteamericanos invertidos en la producción. En términos relativos el municipio de FIM, Coahuila generó el 78\% del empleo que se generó a nivel distrital empleando la misma cantidad de capital invertido en la producción.

Por otro lado, bajo las mismas condiciones de cultivo, así como de mercado, la cantidad mínima que se requiere producir de nuez en promedio para tener una operación viable (punto de equilibrio) fue de 1,10 ton ha ${ }^{-1}$ en promedio para FIM, Coahuila, (1,08 ton ha ${ }^{-1}$ en nogal irrigado por bombeo y 1,74 ton ha- en nogal irrigado por gravedad) mientras en huertos en promedio del Distrito de Riego 017 se obtuvo un punto de equilibrio de 0,60 ton ha ${ }^{-1}$, por lo que tomando en cuenta los rendimientos obtenidos, se observa que tanto a nivel municipal como regional, cubre el punto de equilibrio, lo que indica que los huertos son rentables tanto a nivel municipal como a nivel regional.

Finalmente se analizó un índice de vulnerabilidad crediticia (el cual se obtuvo al dividir el rendimiento físico, entre el punto de equilibrio, ambos en ton ha ${ }^{-1}$ ) que tiene cada uno de los huertos analizados de acuerdo con sus niveles tecnológicos, entendida como la capacidad que tendría un productor para solventar en determinando momento el pago de un crédito para la producción primaria. Se encontró que los huertos bajo riego por bombeo tuvieron un indicador igual a 1,08 $\left(1,08=\left(0,93\right.\right.$ ton $\mathrm{ha}^{-1} / 0,86$ ton ha-1)$)$, lo cual garantiza la devolución del crédito, mientras que los huertos bajo riego por gravedad tuvieron un indicador de $1,74\left(1,74=1,27\right.$ ton $\mathrm{ha}^{-1} / 0,73$ ton $\left.\mathrm{ha}^{-1}\right)$, mismo que señala que al producir 1,27 ton $\mathrm{ha}^{-1}$, los productores de nuez irrigada por agua superficial proveniente de las presas locales, mediante gravedad, están un $74 \%$ arriba de la cantidad mínima de nuez por hectárea $\left(0,73\right.$ ton $\left.\mathrm{ha}^{-1}\right)$ necesaria para empezar a producir ganancias, el indicador a nivel municipal fue 1,10, mientras que los huertos a nivel distrital tuvieron un índice igual a 2,14, lo que posicionaría a los 
productores de FIM en posición desventajosa en relación al productor promedio regional del DR-017 dado por el indicador 2,14. Aunque los dos niveles tecnológicos (riego por bombeo y riego por gravedad) garantizarían la devolución de determinado crédito, el rendimiento generado por la implementación de ese crédito serían diferentes, de allí que la $\mathrm{R} \mathrm{B} / \mathrm{C}$ en cada uno de los casos estuviera marcada por estas diferencias (Tabla, 1 ).

Tabla 1

Indicadores de la productividad del suelo, del agua, del capital y de la fuerza laboral en la producción del cultivo de Nogal pecanero (Carya illinoiensis) en el municipio de Francisco I. Madero, Coahuila.

Cifras monetarias en USD.

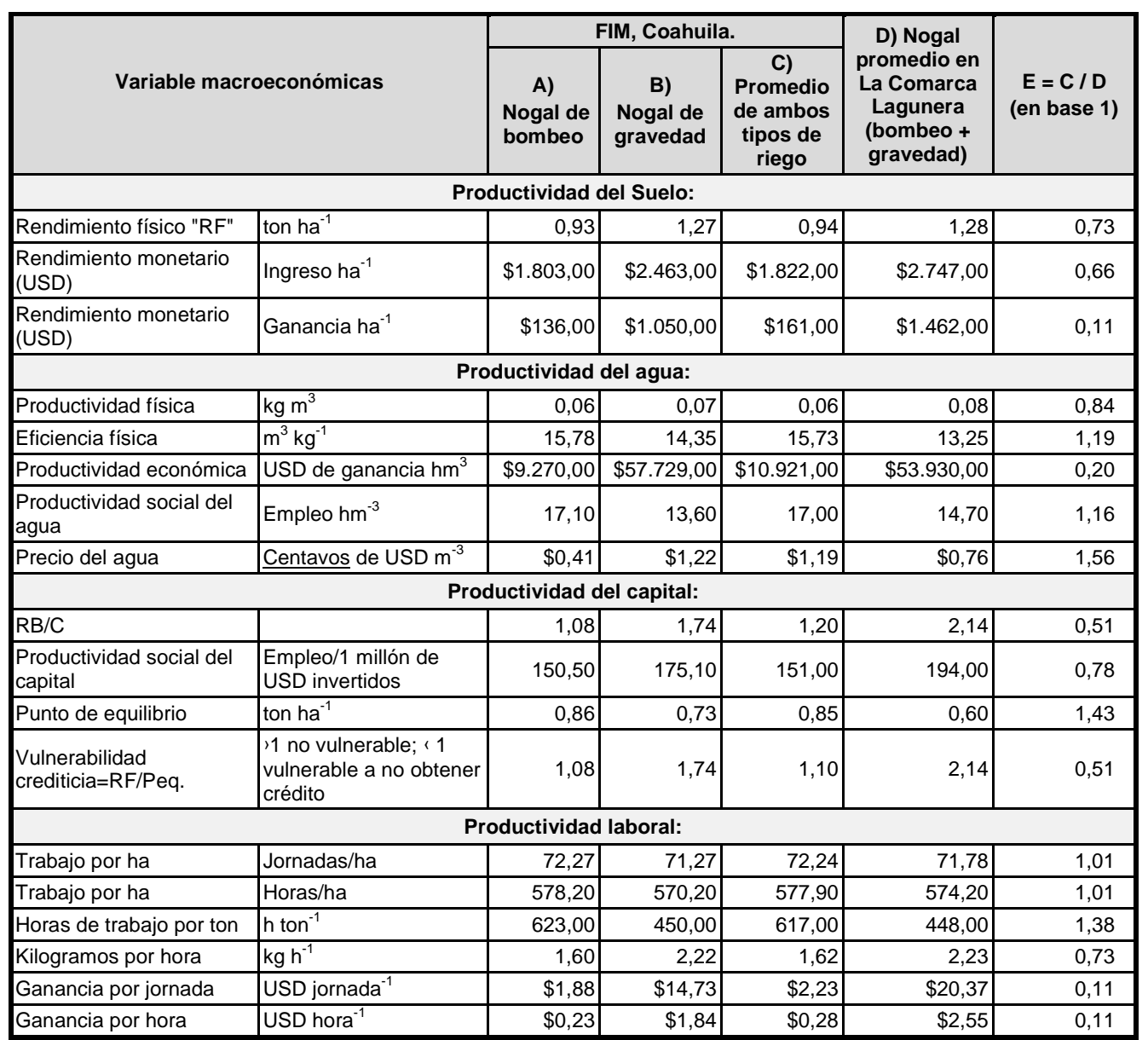

Fuente: Elaboración propia. 


\subsection{Importancia de los indicadores de huella hídrica}

El uso de los indicadores de la $\mathrm{HH}$ física, económica y social permite optimizar el recurso agua mediante su minimización, a la vez que se maximizan objetivos como el Valor Bruto de la Producción (VBP) o las ganancias o el empleo generado en una región agrícola, contribuyendo de esa manera la $\mathrm{HH}$, a hacer sustentable en el largo plazo a la producción agrícola. Ríos et al. (2015) determinaron en primer lugar las HH física, económica y social de todo el patrón agrícola de cultivos forrajeros irrigados mediante agua subterránea en el norte de México, para en un segundo momento, y en base precisamente a la $\mathrm{HH}$, generar escenarios de patrón agrícola diferentes al actual (al que se llamó PAA) caracterizados por la desaparición de ciertos cultivos altamente demandantes de agua para producir un $\mathrm{kg}$ de forraje, o que requerían mucha agua para producir un dólar de ganancia o que necesitaban mucha agua para generar un empleo y en su lugar se proponía la ampliación de la superficie de aquellos forrajes con HH pequeñas, es decir, que usaban poca agua para producir un kg de forraje, o un dólar de ganancia o un empleo. En un tercer momento contrastaron esos escenarios de patrones agrícolas alternativos (PAA1, PAA2) para determinar su impacto en el volumen global de agua consumido, así como en el empleo y la riqueza generados. El PAA se caracterizó por usar $1038 \mathrm{hm}^{3}$ de agua, un VBP de USD1378 millones, un monto regional de ganancias del orden de USD42.9 millones, generar 4975 empleos permanentes y una $\mathrm{RB} / \mathrm{C}$ igual a 1.45. Se determinó que el escenario 1 (PAA1) caracterizado por desaparecer las superficies de forrajes como alfalfa, avena forrajera y zacate ballico o rye grass, y en su lugar sembrar solamente maíz y sorgo forrajeros, así ese escenario del PAA1, si bien reducía el VBP, las ganancias y el empleo en 2.5\%, 11.7\% y 9\% lo cual pareciera ser es "malo", pero, al comparársele contra una reducción del 47\% del volumen anual de agua subterránea extraída del subsuelo (igual a 492 millones de $\mathrm{m}^{3}$ en términos absolutos) resulta un "buen” patrón agrícola, pues permitía reducir la presión que el PAA ejerce sobre el manto freático, ya que anualmente se extraen 1200 millones de $\mathrm{m}^{3}$ mientras que solamente se recargan a ese manto freático un volumen de 450 millones de $\mathrm{m}^{3}$, permitiendo por tanto, que el volumen extraído $\left(546 \mathrm{hm}^{3}\right)$ por el PAA1 tendiese a acercarse al volumen de recarga anual del manto freático.

En el caso de este trabajo, es parte de una serie de estudios sobre las $\mathrm{HH}$ del nogal en el DR017, y una vez se tengan las HH física, económica y social del nogal producido en cada uno de los quince municipios del DR017 y en cada una de sus formas de riego (bombeo tradicional, riegos presurizados altamente tecnificados y riego superficial por gravedad), se elaborarán patrones agrícolas alternativos que redistribuyan geográficamente la producción de nuez en la región, para optimizar el agua de riego a la vez que se maximice el empleo, las ganancias y el VBP. 


\subsection{Productividad del trabajo}

De acuerdo con Dorward (2013), existen otras dos formas de expresar la productividad laboral, para indicadores estructurales, pudiendo ser medida por el valor bruto de la producción generado en relación con el número de personas empleadas y/o por el número de horas trabajadas. La parte inferior de la Tabla 1 contiene los números índice que evalúan la productividad de la fuerza laboral. Los resultados indican que a nivel municipal se invirtieron un total de 617 horas de trabajo por tonelada de nuez (578,2 h ton ${ }^{-1}$ en el nogal irrigado por bombeo y $570,2 \mathrm{~h} \mathrm{ton}^{-1}$ en el nogal irrigado por gravedad), mientras que a nivel distrital se emplearon un total de 574,2 $\mathrm{h} \mathrm{ton}^{-1}$ de nuez, lo que indica que la producción de nuez de FIM, Coahuila es menos productiva, toda vez que empleo más cantidad de horas de trabajo para producir una tonelada de producto, más aún, tanto a nivel de FIM como a nivel de todo el DR-017 existe poca competitividad en la rama nogalera, ya que de acuerdo con Ríos et al. (2016) en el DR-005, en Delicias, Chihuahua, México, principal región productora de nuez en México, se requiere de solamente 72,56 horas de trabajo para producir una tonelada de nuez. Visto de otra forma a nivel municipal, en FIM se generaron $1,62 \mathrm{~kg} \mathrm{~h}^{-1}$ (1,60 $\mathrm{kg} \mathrm{h}^{-1}$ en el nogal irrigado por bombeo y $2,22 \mathrm{~kg} \mathrm{~h}^{-1}$ en el nogal irrigado por gravedad), mientras que a nivel Distrito de Riego se generaron $2,23 \mathrm{~kg} \mathrm{~h}^{-1}$, lo que reafirma lo asentado en el párrafo anterior, en sentido de que los huertos de nogal del municipio de FIM, Coahuila fueron menos productivos al generar solamente el 73\% (el indicador fue 0,73 ) de lo que se generó a nivel distrital.

Se determinó que en promedio los trabajadores adscritos a la producción de nogal en FIM, Coahuila generaron una ganancia de USD 2,23 por jornada trabajada, desglosada en USD 1,88 jornada $^{-1}$ en nogal irrigado por bombeo y USD 14,73 jornada $^{-1}$ en nogal irrigado por gravedad, mientras que a nivel distrital la ganancia obtenida fue de USD 20,37 jornada $^{-1}$, lo que indica que la producción de nogal en FIM, Coahuila en términos de productividad laboral fue menor a la obtenida a nivel regional, ya que la jornada de trabajo invertida en FIM, Coahuila generó apenas el $11 \%$ de la ganancia por jornada de trabajo en comparación a la generada a nivel distrital. Visto de otra forma, mientras a nivel de FIM se generaron USD 0,28 por hora de trabajo (USD $0,23 \mathrm{~h}^{-1}$ en nogal irrigado por bombeo y USD $1,84 \mathrm{~h}^{-1}$ en nogal irrigado por gravedad), en el Distrito de Riego 017, esa misma hora de trabajo produjo una ganancia de USD 2,55, lo que indica que el municipio de FIM, Coahuila fue menos productivo en relación al Distrito de Riego 017, en esa forma de productividad laboral.

La Tabla 2 muestra los costos por hectárea a nivel de los tres niveles de agregación: bombeo, gravedad y todo el nogal de FIM, sin importar si fue producido en bombeo o gravedad. De esa fuente se observa que son dos las fuentes del costo por hectárea: el costo de operación y otros costos. En el primer rubro se incluyen la siembra o plantación. Labores culturales, riego y drenaje, 
dentro del cual se encuentra el subcomponente del costo de extracción del agua si es bombeo o del pago de cuota a la Secretaría de Agricultura si es riego por gravedad), control de plagas, cosecha y diversos (de índole administrativo), mientras que dentro del segundo componente de costos por hectárea se encuentra la depreciación de maquinaria y equipo, renta del suelo, costos financieros y seguro agrícola. Los costos de operación representan el 76,7\% del costo total, mientras que los otros costos suman el 23,3\% restante. Debe observarse que el costo del agua, dentro del total, apenas representa el $10.6 \%$, que en términos absolutos asciende a MX\$ 3.426 (USD 175,85), monto de dinero que permitió la extracción de $14.732 \mathrm{~m}^{3}$ de agua por hectárea para una hectárea promedio sin importar si está siendo irrigada por bombeo o por gravedad $\left(14.634 \mathrm{~m}^{3}\right.$ en bombeo y $18.182 \mathrm{~m}^{3}$ en riego por gravedad), de ahí el tan bajo precio al que el productor paga cada metro cúbico usado de agua: 1,19 centavos de USD, es decir, casi un centavo de dólar por metro cúbico, con mucho, muy inferior al precio del agua reportado por Gleick (2000) de USD 0,05 (5 centavos) por metro cúbico para los agricultores de Estados Unidos de América.

\section{Tabla 2}

Costos de producción por hectárea en el cultivo de nogal (Carya illinoensis) en el DR017, Comarca Lagunera, México

\begin{tabular}{|c|c|c|c|}
\hline $\begin{array}{l}\text { Componentes del costo de producción por } \\
\text { hectárea }\end{array}$ & $\begin{array}{l}\text { Nogal de } \\
\text { Bombeo }\end{array}$ & $\begin{array}{l}\text { Nogal de } \\
\text { Gravedad }\end{array}$ & $\begin{array}{c}\text { Nogal de Bombeo } \\
\text { más Nogal de } \\
\text { Gravedad }\end{array}$ \\
\hline \multicolumn{4}{|l|}{ a) Costos de operación: } \\
\hline 1.- Siembra o plantación & $\$ 2.346,00$ & $\$ 2.342,00$ & $\$ 2.346,00$ \\
\hline 2.- Labores culturales & $\$ 6.635,00$ & $\$ 6.624,00$ & $\$ 6.634,00$ \\
\hline 3.- Riego y drenaje & $\$ 4.362,00$ & $\$ 2.184,00$ & $\$ 4.302,00$ \\
\hline 3.1.- Costo del agua & $\$ 3.483,00$ & $\$ 1.440,00$ & $\$ 3.426,00$ \\
\hline 4.- Control de plagas y enfermedades & $\$ 1.607,00$ & $\$ 1.604,00$ & $\$ 1.607,00$ \\
\hline 5.- Cosecha & $\$ 3.525,00$ & $\$ 3.519,00$ & $\$ 3.525,00$ \\
\hline 6.- Diversos & $\$ 2.994,00$ & $\$ 2.686,00$ & $\$ 2.985,00$ \\
\hline Subtotal costos de operación & $\$ 24.952,00$ & $\$ 20.399,00$ & $\$ 24.825,00$ \\
\hline \multicolumn{4}{|l|}{ b) Otros costos } \\
\hline 7.- Seguro agrícola & $\$ 840,00$ & $\$ 687,00$ & $\$ 835,00$ \\
\hline 8.- Costo financiero & $\$ 1.452,00$ & $\$ 1.201,00$ & $\$ 1.445,00$ \\
\hline 9.- Renta del suelo & $\$ 4.000,00$ & $\$ 4.000,00$ & $\$ 4.000,00$ \\
\hline 10.- Depreciación de maquinaria y equipo & $\$ 1.247,00$ & $\$ 1.247,00$ & $\$ 1.247,00$ \\
\hline Subtotal otros costos & $\$ 7.539,00$ & $\$ 7.135,00$ & $\$ 7.527,00$ \\
\hline COSTO TOTAL (MX\$) & $\$ 32.491,00$ & $\$ 27.534,00$ & $\$ 32.352,00$ \\
\hline COSTO TOTAL (USD) & $\$ 1.668,00$ & $\$ 1.413,00$ & $\$ 1.661,00$ \\
\hline Jornales por hectárea & 72,27 & 71,27 & 72,00 \\
\hline
\end{tabular}

Fuente: Elaboración propia, con base en los costos de producción reportados en el Anuario Estadístico de la Producción Agropecuaria ciclo agrícola 2014, DR-017 Comarca Lagunera, Ciudad Lerdo, Durango, México. 


\section{CONCLUSIONES}

Las ventajas de evaluar la productividad del agua de riego mediante el uso de metodología basada en ecuaciones matemáticas explícitas utilizadas para evaluar las huellas hídricas física, económica y social de Ríos et al. (2015, 2016 y 2016 a) son, en primer lugar la certeza de saber el origen de lo que arroja el modelo de salida en función de los datos de entrada, así como la replicabilidad en otras condiciones diferentes a las de este estudio, algo que no puede lograrse utilizando metodologías implícitas de la huella hídrica.

La asignación del recurso agua por parte de los administradores de recursos, específicamente de los tomadores de decisiones, encargados no solo de asignar sino también decidir la distribución de los volúmenes de agua a usar en el sector agrícola, debe tener como objetivo prioritario el uso sostenido a largo plazo del recurso agua, y para ello deberá cimentarse en el uso de indicadores de productividad física, económica y social del agua de riego, ya que en función de los objetivos exógenos impuestos a la producción agrícola, como la maximización del beneficio social mediante la creación de empleo, o la maximización en la generación de la riqueza, el ahorro de agua a usar en la producción agrícola será el punto de partida que cualquier objetivo, ya que, al minimizar el volumen de agua a la par que se maximizan algunos objetivos, es el único camino para lograr la sustentabilidad agrícola.

\section{REFERENCIAS BIBLIOGRÁFICAS}

DORWARD, A. (2013). "Agricultural labour productivity, food prices and sustainable development impacts and indicators". Food Policy, 39(1), pp. 40-50.

GARCÍA, J. G.; LÓPEZ, F. C.; USAI, D. y VISANI, C. (2013). "Economic Assesment and Socio-Economic Evaluation of Water Use Efficiency in Artichoke Cultivation". Open Journal of Accounting, 2(2), pp. 45-52.

GLEICK. (2000). The World's Water, 2000-2001: The Biennial Report on Freshwater Resources. Washington, DC.: Islan Press, 2000. 335 p.

HOEKSTRA, A.Y. (2009). "Human appropriation of natural capital: a comparison of ecological footprint and water footprint analysis". Ecological Economics, 68, 1963-1974.

HOEKSTRA, A.Y. y CHAPAGAIN, A.K. (2008). Globalization of Water: Sharing the Planet's Freshwater Resources. Oxford, UK: Blackwell Publishing.

HOEKSTRA, A.Y. y CHAPAGAIN, A.K. (2004). Water Footprints of Nations. UNESCOIHE. Institute for Water Education. Value of Water. Research Report Series. Serie 16. Volume 1. Appendices. Netherlands.

HOEKSTRA, A. Y. y HUNG, P.Q. (2005). "Globalization of water resources: international virtual water flows in relation to crop trade". Global Environmental Change, 15, pp. 4556. 
INEGI (2015). Prontuario de información geográfica municipal de los Estados Unidos Mexicanos. FIM, Coahuila de Zaragoza Clave geoestadística 05009. http://www3.inegi.org.mx/sistemas/mexicocifras/datos-geograficos/05/05009.pdf [Último acceso: 16 diciembre del 2016].

MEKONNEN, M. M. y HOEKSTRA, A. Y. (2010). "The green, blue and grey water footprint of farm animals and animal products". 2010. Value of Water Research Report Series No. 48 2010, UNESCO-IHE, Delft, the Netherlands.

MURPHY, D.E. (2003). "In a first, U.S. puts limits on California's thirst". New York Times, 5 January, pp. 1-16.

RÍOS FLORES, J. L.; TORRES MORENO, M.; CASTRO FRANCO, R.; TORRES MORENO, M. A. y RUIZ TORRES, J. (2015). "Determinación de la huella hídrica azul en los cultivos forrajeros del DR-017, Comarca Lagunera, México". Rev. FCA UNCUYO, 47(1), pp. 93-107.

RíOS FlORES, J. L.; TORRES MORENO, M. y TORRES MORENO, M. A. (2016). Productividad agrícola del agua en nogal pecanero del norte de México. Casos: Comarca Lagunera y Delicias, Chihuahua. ISBN: 978-3-639-80166-8. Editorial Académica Española. pp. 37.

RíOS FLORES, J. L.; TORRES MORENO, M.; RUIZ TORRES, J. y TORRES MORENO, M. A. (2016a). "Eficiencia y productividad del agua de riego en trigo (Triticum vulgare) de Ensenada y Valle de Mexicali, Baja California, México". Acta Universitaria, 26(1), pp. 20-29.

SAGARPA (2014). Anuario Estadístico de la Producción Agrícola ciclo agrícola 2014. Lerdo, Durango, México.

SIAP (2014). http://infosiap.siap.gob.mx/aagricola_siap_gb/icultivo/index.jsp

TAKELE, E. y KALLENBACH, R. (2001). "Analysis of the Impact of Alfalfa Forage Production under Summer Water-Limiting Circumstances on Productivity, Agricultural and Growers Returns and Plant Stand". Journal of Agronomy and Crop Science, 187(1), pp. 41-46.

WICHELNS, D. (2001). "The role of 'virtual water' in efforts to achieve food security and other national goals, with an example from Egypt". Agricultural Water Management, 49, pp. 131-151. 
